\title{
Identifying the Direction of Behavioral Dependence in Two-Sample Capture-Recapture Study
}

\author{
Kiranmoy Chatterjee ${ }^{1}$ and Diganta Mukherjee ${ }^{2}$
}

\begin{abstract}
With the possibility of dependence between the sources in a capture-recapture type experiment, identification of the direction of such dependence in dual system of data collection is vital. This has a wide range of applications, including in the domains of public health, official statistics and social sciences. Owing to the insufficiency of data for analyzing a behavioral dependence model in dual system, our contribution lies in the construction of several strategies that can identify the direction of underlying dependence between the two lists in the dual system, that is, whether the two lists are positively or negatively dependent. Our proposed classification strategies would be quite appealing for improving the inference as evident from recent literature. Simulation studies are carried out to explore the comparative performance of the proposed strategies. Finally, applications on three real data sets from various fields are illustrated.
\end{abstract}

Key words: Classification; direction of behavioral dependence; human population; randomized rule; recapture probability.

\section{Introduction and Motivation}

Estimation of the size of a given population is an important statistical concern that has vast application in the field of public health, population studies and animal abundance. In practice, it is mostly impossible to count all the individuals in a population accurately by any attempt, especially when the population is large enough or very hard to reach. As a remedy, more than one attempt is carried out independently and the population size $(N)$ is estimated by matching the available (two or more) lists of information. This kind of data structure is known as a multiple-record system, which is equivalent to the capturerecapture system popularly relevant to abundance of animal population. However, in the context of a closed human population, use of more than two sources of information is uncommon in the official registration systems of most countries. When two attempts have been made to estimate the $N$ in capture-recapture format, then the resulting data structure is known as a dual-record system (DRS), which is presented in Table 1. Estimation of census coverage error (Gerritse et al. 2017; Chatterjee and Mukherjee 2016a), epidemiological events (Iñigo et al. 2003; Granerod et al. 2013), size of hard-to-count

\footnotetext{
1 Department of Statistics, Bidhannagar College, Salt Lake City, Kolkata, 700064, India. Email: kiranmoy07@gmail.com

2 Sampling and Official Statistics Unit, Indian Statistical Institute, Kolkata, 700108, India. Email: diganta@isical.ac.in

Acknowledgments: Authors gratefully acknowledge the partial financial support by the research fellowship award (Office Order No. DS/JSTK-CC-0020F Dated 11 July, 2013) received by the first author from Indian Statistical Institute, India during his attachment with the institute.
} 
Table 1. Data structure in Dual-Record System (DRS).

List 2

\begin{tabular}{lccc}
\cline { 2 - 4 } List 1 & In & Out & Total \\
\hline In & $x_{11}$ & $x_{10}$ & $x_{1}$. \\
Out & $x_{01}$ & $x_{00}$ & $x_{0}$. \\
\hline Total & $x_{\cdot 1}$ & $x_{\cdot 0}$ & $x . .=N$ \\
\hline
\end{tabular}

population (Ruiz et al. 2016) are the primary applications of DRS for human population. ChandraSekar and Deming (1949) proposed homogeneous post-stratification of the data obtained in DRS structure in order to reduce heterogeneity with respect to the capture probabilities among individuals. This proposal has been commonly applied in most of the official statistics, as well as in epidemiological data sets (Eckberg 2000; Iñigo et al. 2003). Bohning et al. (2017) discussed some recent developments of the applications of various capture-recapture models in the arena of epidemiology, medical and social science.

After the construction of such mutually exclusive post-strata, which are within homogeneous but between heterogeneous, relevant statistical models for DRS can be analyzed for each of those post-strata. Model $M_{t}$ (Otis et al. 1978), equivalent to the Petersen Model (Wolter 1986), has received much attention among practitioners because of its simplicity and identifiability in the vicinity of DRS for a human population. In Table 1, $x_{1}, x_{x_{1}}$ and $x_{11}$ refer to the number of individuals present in the first list (List 1), second list (List 2) and their common list, respectively. Following the underlying assumption of list-independence between the two lists, the estimator of $N$ based on the model $M_{t}$ is found to be $\frac{x_{1} \cdot x_{1}}{x_{11}}$ (Wolter 1986; Chatterjee and Mukherjee 2016b). This estimator is popularly known as the Petersen estimator in epidemiology, or the ChadraSekar-Deming estimator in the domain of demography or population studies. More details on this model, including various likelihood based estimating approaches, can be found in Chatterjee and Mukherjee (2016b).

However, the assumption of list-independence may seriously mislead in many situations for human populations. Several methodologists and practitioners (ChandraSekar and Deming 1949; Greenfield 1975; El-Khorazaty 2000; Jarvis et al. 2000; Chao et al. 2001) argued that the list-independence assumption may not be justifiable in reality. An efficient brief review was done by Brittain and Bohning (2009) on the various methods available by relaxing the independence assumption and associated comparative study was undertaken in DRS context. The assumption of list-independence is often violated due to the presence of behavioral response variation at the time of a second capture attempt in DRS. An individual who is enlisted in List 1 may be more likely to also be included in List 2 than the individual who has not been enlisted in List 1. Hence, the corresponding population is treated as recapture prone. This kind of behavioral connection at the second time attempt is commonly encountered in epidemiological (Chao et al. 2001; Granerod et al. 2013) and demographic (Bell 1993; Griffin 2014) studies. Otherwise, in reverse cases, populations become recapture averse, for example hard-to-count population, drug addicted population. An interesting attempt to judge the effect of violation of the listindependence in this context was done by Gerritse et al. (2017). These kinds of changes in 
the behavior of an individual, while he/she attempts to be included in List 2, is grossly known as behavioral response variation (Wolter 1986; Chatterjee and Mukherjee 2018). O'Connell and Pollock (1992) introduced a strategy using demographic co-variates for partitioning a population in terms of direction of behavioral dependence. When both the time variation and behavioral response variation act together, model $M_{t b}$, to be defined later, can be treated as the most general and suitable statistical model for analyzing capture-recapture data under homogeneity. Gosky and Ghosh (2011) showed the appropriateness of this model among all the capture-recapture models proposed in Otis et al. (1978).

Now we state some basic notation before proceeding further. Let $p_{i j}$ be the probability attached to each individual to be included in the count $x_{i j}$ in $(i, j)$ th cell of Table 1 , where $i, j \in\{0,1, \cdot\}$. In addition we denote

$\operatorname{Pr}($ an individual is captured in List $2 \mid \mathrm{s} /$ he is listed in List 1$)=\frac{p_{11}}{p_{1}}=c$,

$\operatorname{Pr}$ (an individual is captured in List $2 \mid \mathrm{s} /$ he is not listed in List 1$)=\frac{p_{01}}{1-p_{1}}=p$, following Wolter (1986). In this article, we consider $c \neq p$ which refers a violation of independence between the two lists. Thus, there always exists some constant $\phi(>0)$ such that $c=\phi p$. This $\phi$ is termed as behavioral response effect. Note that $\phi>1$ (equivalently, $c>p$ ) refers to positive association between the two sources and the associated population is said to be recapture prone and $\phi<1$ (equivalently, $c<p$ ) refers to negative association and the associated population is said to be recapture averse. A capture-recapture model with time variation and behavioral response variation, denoted as $M_{t b}$, incorporates behavioral dependence between lists. However, model $M_{t b}$ suffers from a problem in DRS, as $\phi$ or $p$ is not estimable separately, but their product $\phi p=c$ is. This unidentifiability of the realistic model $M_{t b}$ in DRS is discussed in Chao et al. (2000) and Chatterjee and Mukherjee (2016c).

One important aspect of the model $M_{t b}$ is that if some proper knowledge on the direction of $\phi$ is available, then it might help to draw a reasonably good inference on the $N$, as evident from literature (Nour 1982; Chatterjee and Mukherjee 2016c, 2018). Particularly, if underlying $\phi$ for a population is correctly known to be greater than 1 (or less than 1), then uncertainty on $\phi$ will be reduced, since the domain of $\phi$ shrinks to $(1, \infty)$ (or $(c, 1))$, where $c$ is the recapture probability of an individual, defined earlier. Hence, one can expect that inference would likely be better if that available knowledge is used. This issue has been proved empirically in Chatterjee and Mukherjee (2016c, 2018). For example, in a demographic study or homicide death study, two sources (or, lists) are commonly positively depended on, which leads to a high estimate of $c$. On the one hand, in economic surveys, people often want to avoid to be enlisted repeatedly in both the lists, and that leads to low estimate of the recapture probability $c$. Therefore, one may intuitively suggest that a given population is recapture prone if the maximum likelihood estimate of $c$, denoted by $\hat{c}$, is very close to its upper bound 1 . On the other hand, if $\hat{c}$ is very close to its lower bound 0 , then the associated population would be recapture averse with high probability. Giving an idea about the possible direction of $\phi$ is always a challenging job if $\hat{c}$ is neither close to 1 , nor close to 0 . As per our knowledge, no strategy has been developed yet to infer the direction of $\phi$ from capture-recapture data only. Therefore, in this article, our aim is to develop some 
classification strategies with which a given population can be identified as recapture prone, recapture averse, or list-independent with high probability in connection with DRS.

In this article, based on various considerations, we propose a large number of classification strategies in Section 2 to identify the direction of list-dependency in DRS. Performance of the proposed strategies are thoroughly investigated with simulated data in Section 3. Based on their performances, a subset of the proposals are applied to three real data sets from various fields of application including demography, crime statistics and economics in Section 4. We illustrate our classification strategies through inference about the direction of inherent dependence in DRS corresponding to each of the three data sets. Finally, in concluding Section 5, we discuss the implications, advantages and possible extensions of the proposed classification strategies.

\section{Proposed Classification Strategies}

In this section, we propose strategies to identify whether individuals belonging to a given population are recapture prone or recapture averse or their inclusion statuses in DRS are list-independent. To formulate such strategies, we need to impose an assumption on a parameter of the model $M_{t b}$. For the present context, we consider that the conditional probability $p \geq \pi$, where a value of $\pi$ can be chosen. Note that this assumption allows a larger interval of possible values of $p,[\pi, 1)$, as one chooses a smaller $\pi$. We will discuss this choice later. For a justification of this, we refer to a parallel assumption adopted in Nour (1982) for estimating total number of vital events (e.g., birth, death) where the capture probability in each of the two lists (i.e., $p_{1}$. and $p_{._{1}}$ ) is assumed to be greater than 0.5. Now, from the definition of $p$, stated in Section 1, one can write that

$$
p=\frac{p_{01}}{1-p_{1}}=\frac{x_{01}}{N-x_{1}}
$$

since DRS satisfy $N p_{i j}=x_{i j}$ for all $i, j \in\{0,1, \cdot\}$. The assumption of $p \geq \pi$ can be rewritten as $(c-\phi \pi) \geq 0$, since $c=\phi p$. By virtue of the mle, $\hat{c}=\left(x_{11} / x_{1}\right.$.) being consistent, as well as an efficient estimator of $c$, we replace $c$ by $\hat{c}$ and use the approximate relation $\phi=\hat{c} p^{-1}$ for sufficiently large $N$. Therefore, as $N \geq x_{0}$, where $x_{0}$ denotes the total number of distinct individuals counted in DRS,

$$
\begin{gathered}
\phi=\hat{c} p^{-1}=\frac{x_{11}}{x_{1} \cdot} \frac{N-x_{1} .}{x_{01}} \geq \frac{x_{11}}{x_{1}} . \\
\text { or }\left(x_{1} \cdot \phi-x_{11}\right) \geq 0 .
\end{gathered}
$$

Thus, using the inequalities in Equation (1) and $(\hat{c}-\phi \pi) \geq 0$, obtained earlier with the consideration of approximation of $c$ by $\hat{c}$, we consider the (composite) inequality

$$
\frac{(\hat{c}-\phi \pi)\left(x_{1} . \phi-x_{11}\right)}{x_{\cdot 1} \phi} \geq k
$$

for $x_{\cdot 1}>0$, where $k$ is some non-negative real number. The choice of $k$ will be explained later. Hence, the above inequality may be expressed as 


$$
\begin{gathered}
\phi^{2}+\phi \frac{k x_{\cdot 1}(\pi+1) x_{11}}{\pi x_{1} \cdot}+\hat{c}^{2} \pi^{-1} \leq 0 \\
\text { or, }\left(\phi-\phi_{0}\right)\left(\phi-\phi_{1}\right) \leq 0
\end{gathered}
$$

where $\phi_{0}$ and $\phi_{1}$ are two real roots of the quadratic equation corresponding to (2), which are functions of the unknown $k$ and satisfy $\phi_{0}+\phi_{1}=\frac{(\pi+1) x_{11}-k x_{.1}}{\pi x_{1}}$ and $\phi_{0} \phi_{1}=\hat{c}^{2} \pi^{-1}$ with $\phi_{0} \leq \phi \leq \phi_{1}$ from Equation (3). In fact, the two roots are positive real valued under some condition on $k$. All the mathematical proofs and justifications on the nature of the two said roots of $\phi$ are rigorously discussed in the Appendix (Section 6). Since the arithmetic mean of any two positive real numbers is always greater than or equal to their geometric mean, therefore one will have

$$
k \leqslant \frac{x_{11}}{x_{\cdot 1}}(1-\sqrt{\pi})^{2},
$$

equality holds only when $\phi_{0}=\phi_{1}=\phi=\hat{c} \pi^{-1 / 2}$. From the Appendix we know that the above upper bound of $k$ is the necessary and sufficient condition for both the roots $\phi_{0}$ and $\phi_{1}$ to be positive real-valued. In addition, as $k \geq 0$ holds under the assumption that $p \geq \pi$, the values of $\phi_{0}$ and $\phi_{1}$ corresponding to the lower bound of $k$ are $\hat{c}$ and $\hat{c} \pi^{-1}$ respectively. Furthermore, the root $\phi_{0}$ is a monotonically increasing function of $k$, while $\phi_{1}$ is decreasing in $k$. This implies

$$
\hat{c} \leq \phi_{0} \leq \hat{c} \pi^{-1 / 2}
$$

and

$$
\hat{c} \pi^{-1 / 2} \leq \phi_{1} \leq \hat{c} \pi^{-1}
$$

Proposals. By combining the two above inequalities for the two roots $\phi_{0}$ and $\phi_{1}$, we have

$$
\hat{c} \leq \phi \leq \hat{c} \pi^{-1} .
$$

Now, arithmetic (A.M.), geometric (G.M.) and harmonic (H.M.) means of the two limits of the above interval are, $\frac{(1+\pi) \hat{c}}{2 \pi}, \hat{c} \pi^{-1 / 2}$ and $\frac{2 \hat{c}}{1+\pi}$, respectively. We want to consider a reasonably moderate value of $k$ to allow the possibility of a small value of $\pi$. As discussed earlier, $\phi_{0}$ and $\phi_{1}$ are respectively increasing and decreasing functions of $k$, we consider $\phi_{0} \geq$ H.M. to accommodate such reasonable value of $k$ and it automatically implies $\phi_{1} \leq$ A.M. as $\phi_{0} \phi_{1}=\hat{c}^{2} \pi^{-1}$. Since $\phi_{0} \leq \phi \leq \phi_{1}$, one would therefore readily have H.M. $\leq \phi$ $\leq$ A.M., that is,

$$
\frac{2 \hat{c}}{1+\pi} \leq \phi \leq \frac{(1+\pi) \hat{c}}{2 \pi}
$$

We call this proposed interval for $\phi$ as Proposal 1. One may further propose tighter bounds for the classification strategies as per the following reasoning.

Proposal 2: As $\hat{\mathrm{c}} \pi^{-1 / 2} \leq \phi_{1}$, we may propose the bound for $\phi$ as H.M. $\leq \phi \leq$ G.M., since G.M. is the smallest upper bound for $\phi$. So,

$$
\frac{2 \hat{c}}{1+\pi} \leq \phi \leq \hat{c} \pi^{-1 / 2} \text {. }
$$

One can alternatively propose, 
Proposal 3: As $\phi_{0} \leq \hat{\mathrm{c}} \pi^{-1 / 2}$, we may propose the bound for $\phi$ as G.M. $\leq \phi \leq$ A.M., since G.M. is the greatest lower bound for $\phi$. So,

$$
\hat{c} \pi^{-1 / 2} \leq \phi \leq \frac{(1+\pi) \hat{c}}{2 \pi} .
$$

Now, we present classification rules to identify the direction of behavioral dependence among individuals in a population. Three alternative rules for each of the above three proposals are designed to indicate whether underlying $\phi$ is greater than 1 (i.e., proneness), less than 1 (i.e., aversion) or very close to 1 (i.e. list-independence). Let us consider that $\phi_{l}$ and $\phi_{u}$ respectively are the lower and upper tolerance limits of $\phi$, equidistant from 1 , beyond which one may say that underlying DRS is list-dependent. For example, if we consider $5 \%$ tolerance, then $\left(\phi_{l}, \phi_{u}\right)=(0.95,1.05)$. We outline the classification strategies in detail for Proposal 1 based on the bounds in Equation (4), corresponding to Proposal 1. The variations for other proposals are analogously defined, based on the bounds in Equations (5) and (6).

Rule 1L. We set the lower bound of the interval, $\frac{2 \hat{c}}{1+\pi} \leq \phi \leq \frac{(1+\pi) \hat{c}}{2 \pi}$, that is, $\frac{2 \hat{c}}{1+\pi}$ as a threshold to infer about the direction of dependence. So, if $\frac{2 \hat{c}}{1+\pi}>\phi_{u}$ we say the population is recapture prone. Again if $\frac{2 \hat{c}}{1+\pi}<\phi_{l}$, we say the population is recapture averse. Further, the population will be termed as list-independent if $\phi_{l} \leq \frac{2 \hat{c}}{1+\pi} \leq \phi_{u}$. This lower bound technique may be conservative as it has a tendency towards indicating recapture aversion (see Section 3 ).

Analogously, if we replace the threshold in Rule $1 L$ by the upper bound of the interval, we can define Rule $1 U$. Note that Rule $I U$ may be conservative towards recapture proneness (see Section 3).

Admitting results of the previous classification rules, now we propose a randomized rule, similar to the statistical hypothesis test rule (as in the Neyman-Pearson tradition), that will safeguard the decision rule from possible threats of bias towards recapture proneness or aversion. Here, the randomized decision is taken when the tolerance limits $\phi_{l}$ or $\phi_{u}$ lie in the interval (4).

Rule 1R. We consider the following steps involving randomized decisions to infer about the behavioral classification of a given population.

Step 1: Carry out a randomized trial based on a Bernoulli r.v., say $X p$, with the following probability function in favour of recapture proneness of the given population.

$$
\psi_{p}(\hat{c})=\left\{\begin{array}{lll}
1 & \text { if } & \frac{2 \hat{c}}{1+\pi}>\phi_{u} \\
\delta_{p} & \text { if } & \frac{2 \hat{c}}{1+\pi} \leq \phi_{u}<\frac{(1+\pi) \hat{c}}{2 \pi} \\
0 & \text { if } & \frac{(1+\pi) \hat{c}}{2 \pi} \leq \phi_{u}
\end{array}\right.
$$

where

$$
\delta_{p}=\max \left\{0,1-\left(\phi_{u}-\frac{2 \hat{c}}{1+\pi}\right) /\left(\frac{(1+\pi) \hat{c}}{2 \pi}-\frac{2 \hat{c}}{1+\pi}\right)\right\}
$$


Step 2: If the given population is not found to be recapture prone in Step 1 that is, if $X_{p}$ is not observed to be 1 , carry out another randomized trial based on Bernoulli r.v., say, $X_{a}$ with the following probability function in favor of recapture aversion.

$$
\psi_{a}(\hat{c})=\left\{\begin{array}{lll}
1 & \text { if } & \frac{(1+\pi) \hat{c}}{2 \pi}<\phi_{l} \\
\delta_{a} & \text { if } & \frac{2 \hat{c}}{1+\pi} \leq \phi_{l} \leq \frac{(1+\pi) \hat{c}}{2 \pi} \\
0 & \text { if } & \frac{2 \hat{c}}{1+\pi}>\phi_{l},
\end{array}\right.
$$

where

$$
\delta_{a}=\left(\phi_{l}-\frac{2 \hat{c}}{1+\pi}\right) /\left(\frac{(1+\pi) \hat{c}}{2 \pi}-\frac{2 \hat{c}}{1+\pi}\right)
$$

Step 3: If the given population is not found to be recapture averse in Step 2 that is, if $X_{a}$ is observed to be 0 , the given population is classified as list-independent.

It is to be noted that when the above probability $\psi_{p}(\hat{c})\left(\psi_{a}(\hat{c})\right)$ in Step 1 (Step 2$)$ is neither 1 nor 0 , one has to perform a Bernoulli experiment with probability of recapture proneness (recapture aversion) equal to $\delta_{p}\left(\delta_{a}\right)$, in order to classify whether a given population is recapture prone (averse).

As we have defined Rules $1 L, I U$ and $1 R$ for Proposal 1, one can analogously define Rules $2 L, 2 U$ and $2 R$ for Proposal 2 based on the bounds in Equation (5). Similarly, Rules $3 L, 3 U$ and $3 R$ can be defined for Proposal 3 based on the bounds in Equation (6). We omit the details and proceed to an empirical evaluation of these rules.

The probabilities for considering an individual as recapture prone $(R P)$, recapture averse (RA), or list-independent $(L I)$ can be obtained effectively by computing the probabilities $\operatorname{Pr}\left(X_{p}=1\right), \operatorname{Pr}\left(X_{a}=1, X_{p}=0\right)$ and $\operatorname{Pr}\left(X_{a}=0, X_{p}=0\right)$ respectively. These probabilities are computed based on the asymptotic normality of the mle $\hat{c}$. These probabilities, corresponding to the randomized rule under proposal 2 (i.e., Rule $2 R$ ), are presented in the following theorem and the associated proof is sketched in the Appendix.

Theorem 1. For a large population, probabilities for considering an individual to be recapture prone, recapture averse or list-independent under Rule $2 \mathrm{R}$ respectively, are as follows:

$$
\begin{aligned}
\operatorname{Pr}(R P)= & 1-\left[\delta_{p} F\left(\phi_{u} \sqrt{\pi}\right)-\left(1-\delta_{p}\right) F\left(\frac{(1+\pi) \phi_{u}}{2}\right)\right], \\
\operatorname{Pr}(R A)= & \delta_{a} F\left(\frac{(1+\pi) \phi_{l}}{2}\right)+\left(1-\delta_{a}\right) F\left(\phi_{l} \sqrt{\pi}\right) \quad \text { if } \quad \frac{(1+\pi) \phi_{l}}{2}<\phi_{u} \sqrt{\pi} \\
& \left(1-\delta_{a}\right) F\left(\phi_{l} \sqrt{\pi}\right)+\delta_{a}\left(1-\delta_{p}\right) F\left(\frac{(1+\pi) \phi_{l}}{2}\right)+\delta_{a} \delta_{p} F\left(\phi_{u} \sqrt{\pi}\right) \\
& \text { if } \frac{(1+\pi) \phi_{l}}{2}>\phi_{u} \sqrt{\pi}
\end{aligned}
$$




$$
\begin{aligned}
\operatorname{Pr}(L I)= & {\left[\delta_{p} F\left(\phi_{u} \sqrt{\pi}\right)+\left(1-\delta_{p}\right) F\left(\frac{(1+\pi) \phi_{u}}{2}\right)\right] } \\
& -\left[\delta_{a} F\left(\frac{(1+\pi) \phi_{l}}{2}\right)-\left(1-\delta_{a}\right) F\left(\phi_{l} \sqrt{\pi}\right)\right] \text { if } \frac{(1+\pi) \phi_{l}}{2}<\phi_{u} \sqrt{\pi}, \\
& {\left[\delta_{p}\left(1-\delta_{a}\right) F\left(\phi_{u} \sqrt{\pi}\right)+\left(1-\delta_{p}\right) F\left(\frac{(1+\pi) \phi_{u}}{2}\right)\right] } \\
& -\left[\delta_{a}\left(1-\delta_{p}\right) F\left(\frac{(1+\pi) \phi_{l}}{2}\right)+\left(1-\delta_{a}\right) F\left(\phi_{l} \sqrt{\pi}\right)\right] \text { if } \frac{(1+\pi) \phi_{l}}{2}>\phi_{u} \sqrt{\pi},
\end{aligned}
$$

where $F(\cdot)$ refers the cumulative distribution function of normal variate $\hat{c}$ with asymptotic mean and variance are $c$ and $\sigma_{\hat{c}^{2}}$, respectively.

Theorem 1 is useful for applied work in that it provides a quite important and reasonably simple empirical strategy for detecting behavioral dependence without any need for additional information. All calculations are made here under the normality assumption that is satisfied for reasonably large populations. Note that for some configuration of $\mathrm{p}_{i j}^{\prime} s$ (as defined in the introduction), the boundary constraints in the probability calculations may become binding. In such cases, the conclusion from Theorem 1 would be approximate in nature. Apart from such cases, the strategy will work well. A graphical comparative study between the three probabilities computed in Theorem 1 is carried out in Subsection 3.2.

\section{Simulation Study}

\subsection{Evaluation of Classification Rules}

In this section, we perform an extensive simulation study for comparing the performances of the proposed classification strategies. Let us consider ten simulated populations, comprising three populations for each of the three absolute difference values, $(0.1,0.15$, 0.20 ), between $p_{1}$. and $p_{\cdot 1}$ and one additional population with very high capture probabilities $\left(p_{1} \cdot=0.95, p_{\cdot 1}=0.85\right)$. Further, we also consider three values of $\phi$, viz. 1.50, 0.60, 1.00, in order to represent three situations of behavioral dependence, $(i)$ recapture proneness, (ii) recapture aversion, and (iii) list-independence, respectively. These simulated populations for each of three said $\phi$ values together encompass all possible combinations with true population size, $N=1,000$. The compositions of the simulated populations are shown in Table 2. The true value of the parameter $c$, calculated using the given $p_{1 .}, p_{\cdot 1}$ and $\phi$, is also presented corresponding to each of the ten simulated populations. Tables 3,4 and 5 present the performance evaluation of the classification strategies based on Proposals 1, 2 and 3, respectively, developed in Section 2, in terms of correct classification rate (CCR) of the underlying directional nature of $\phi$. CCR is presented in percentage (\%) after computing the number of correct classifications out of 5,000 replications.

From the simulation analysis we observed that $\pi=1 / 3,1 / 4$ have overall better results across the three proposals (1,2 and 3) and three dependence situations (proneness, aversion and list-independence). Hence, we make a suggestion for choosing $\pi=1 / 3$ or 
Table 2. Compositions of simulated populations with $N=1,000$.

\begin{tabular}{|c|c|c|c|c|c|c|c|}
\hline \multirow[b]{3}{*}{$p_{1}$} & \multirow[b]{3}{*}{$p \cdot 1$} & \multirow{2}{*}{\multicolumn{2}{|c|}{$\begin{array}{c}\text { Recapture prone } \\
\qquad \phi=1.50\end{array}$}} & \multirow{2}{*}{\multicolumn{2}{|c|}{$\begin{array}{c}\text { Recapture averse } \\
\phi \phi=0.60\end{array}$}} & \multirow{2}{*}{\multicolumn{2}{|c|}{$\begin{array}{c}\text { Causally independent } \\
\phi \phi=1.00\end{array}$}} \\
\hline & & & & & & & \\
\hline & & Population & $c$ & Population & $c$ & Population & $c^{*}$ \\
\hline 0.50 & 0.60 & $\mathrm{P} 1$ & 0.720 & A1 & 0.450 & I1 & 0.60 \\
\hline 0.70 & 0.60 & P2 & 0.667 & A2 & 0.500 & I2 & 0.60 \\
\hline 0.70 & 0.80 & P3 & 0.889 & A3 & 0.667 & I3 & 0.80 \\
\hline 0.45 & 0.60 & P4 & 0.735 & A4 & 0.439 & I4 & 0.60 \\
\hline 0.70 & 0.55 & P5 & 0.611 & A5 & 0.458 & I5 & 0.55 \\
\hline 0.80 & 0.65 & P6 & 0.696 & A6 & 0.574 & I6 & 0.65 \\
\hline 0.45 & 0.65 & P7 & 0.796 & A7 & 0.476 & I7 & 0.65 \\
\hline 0.75 & 0.55 & P8 & 0.600 & A8 & 0.471 & I8 & 0.55 \\
\hline 0.85 & 0.65 & P9 & 0.684 & A9 & 0.591 & I9 & 0.65 \\
\hline 0.95 & 0.85 & P10 & 0.864 & A10 & 0.823 & I9 & 0.85 \\
\hline
\end{tabular}

*Under list-independence, that is, $\phi=1, c=p \cdot 1$

1/4 based on empirical performance. It is noted that the upper bound for Proposal 2 is same as the lower bound for Proposal 3 and their union is the bounds for Proposal 1. Hence, we first compare Proposals 2 and 3, which are based on disjoint intervals. By construction, Proposal 2 (Proposal 3) goes against the recapture prone (averse) conclusions for the populations. Thus, comparison on the basis of performances in case of truly listindependent populations will be relatively unbiased. On the basis of empirical performance, Proposal 2 is found to be better than Proposal 3. In addition, it is observed across the simulated populations that Proposal 2 also performs better than Proposal 1 . Overall, we can conclude that Proposal 2 outperforms Proposal 1 which is indeed better than Proposal 3. Choice of the middle values for $\pi$ (e.g., 1/3, 1/4) are better than the extreme values (e.g., 2/5, 1/5). Henceforth, for real data analysis, we prescribe the use of Proposal 2 or 1 with $\pi$ values in the range of $\{1 / 3,1 / 4\}$.

While comparing the rules $\mathrm{L}, \mathrm{U}$ and $\mathrm{R}$ over the chosen proposals and the values for $\pi$, we see that rules $\mathrm{R}$ have a more balanced performance in terms of CCR than that of $\mathrm{L}$ and $\mathrm{U}$ rules. Performance of rule $\mathrm{R}$ for the best choice of proposal (i.e., Proposal 2) would be better understood through further analysis sketched in the next subsection.

\subsection{Performance Study of Rule $2 R$}

Graphical analysis of the probabilities computed based on the result in Theorem 1 for the randomized classification Rule $2 R$ is presented in Figure 1.

Here we consider the same combinations of $\left(p_{1}, p_{\cdot 1}\right)$, which are considered in Table 2 in Subsection 3.1 except the 10th combination $(0.95,0.85)$, with varying $\phi$ values over the domain $(0.40,2.00)$. In general, the performance of Rule $2 R$ is seen to be quite good, except for a few combinations of $\left(p_{1}, p_{\cdot 1}\right)$ values. Overall, we can see that our theoretical intuition put forth in the discussion after Theorem 1 and the findings in Subsection 3.1 are carried over here. 


\begin{tabular}{|c|c|c|c|}
\hline 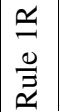 & 苻 & 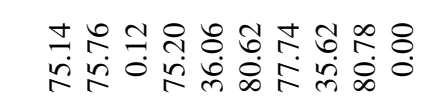 & 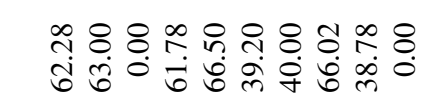 \\
\hline $\mid \begin{array}{l}\supseteq \\
\stackrel{\Xi}{\Xi} \\
\simeq\end{array}$ & 苻 & 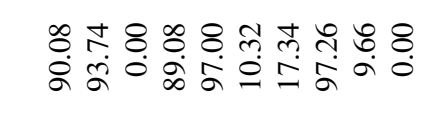 & 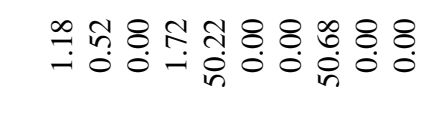 \\
\hline $\mid \begin{array}{l}\exists \\
\stackrel{\Xi}{\vec{\Sigma}}\end{array}$ & 艺 & 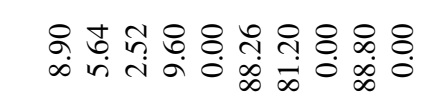 & 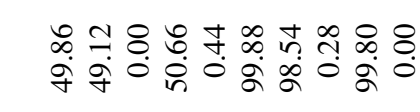 \\
\hline & 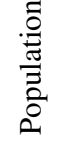 & 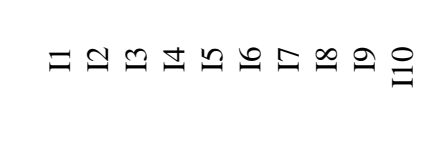 & $\Xi \cong \cong \pm \curvearrowleft ュ ニ \cong の$ \\
\hline 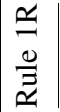 & 늠 & 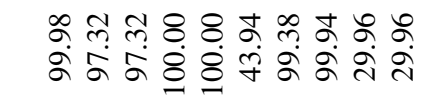 & 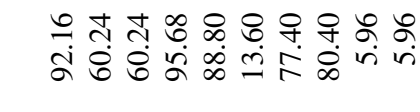 \\
\hline 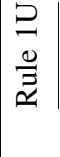 & 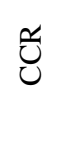 & 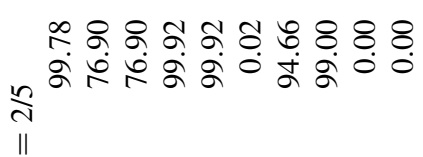 & 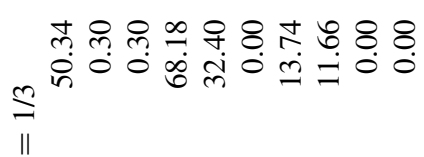 \\
\hline $\mid \begin{array}{l}\exists \\
\frac{0}{\vec{\Xi}}\end{array}$ & $\stackrel{\overbrace{}}{0}$ & 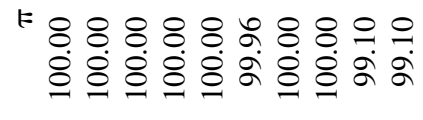 & 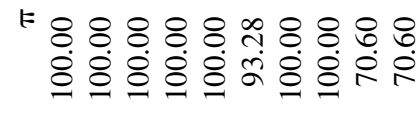 \\
\hline & 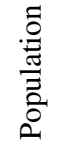 & 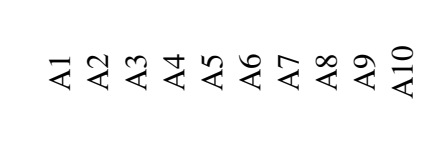 & 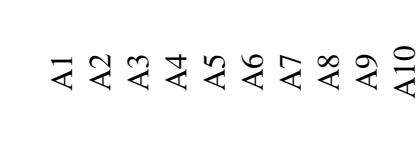 \\
\hline 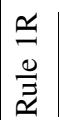 & ठ্ঠ & 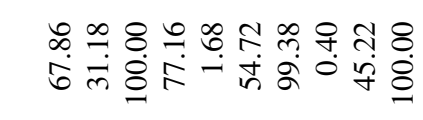 & 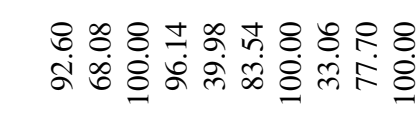 \\
\hline 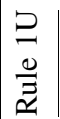 & ֶㅝ & 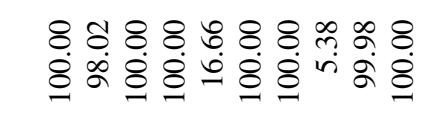 & 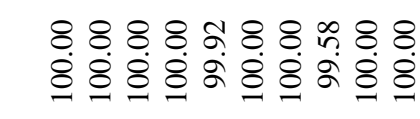 \\
\hline $\mid \begin{array}{l}\exists \\
\stackrel{0}{\vec{\Omega}}\end{array}$ & ठ্ & 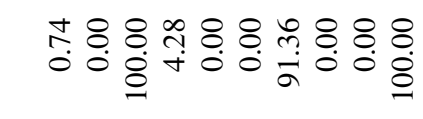 & 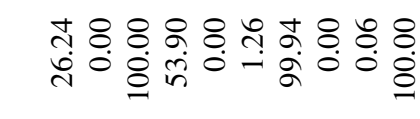 \\
\hline & 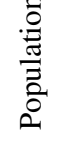 & 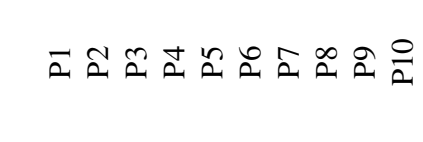 & 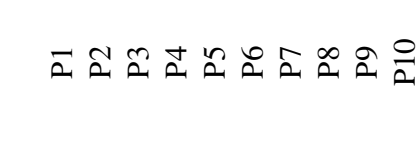 \\
\hline
\end{tabular}




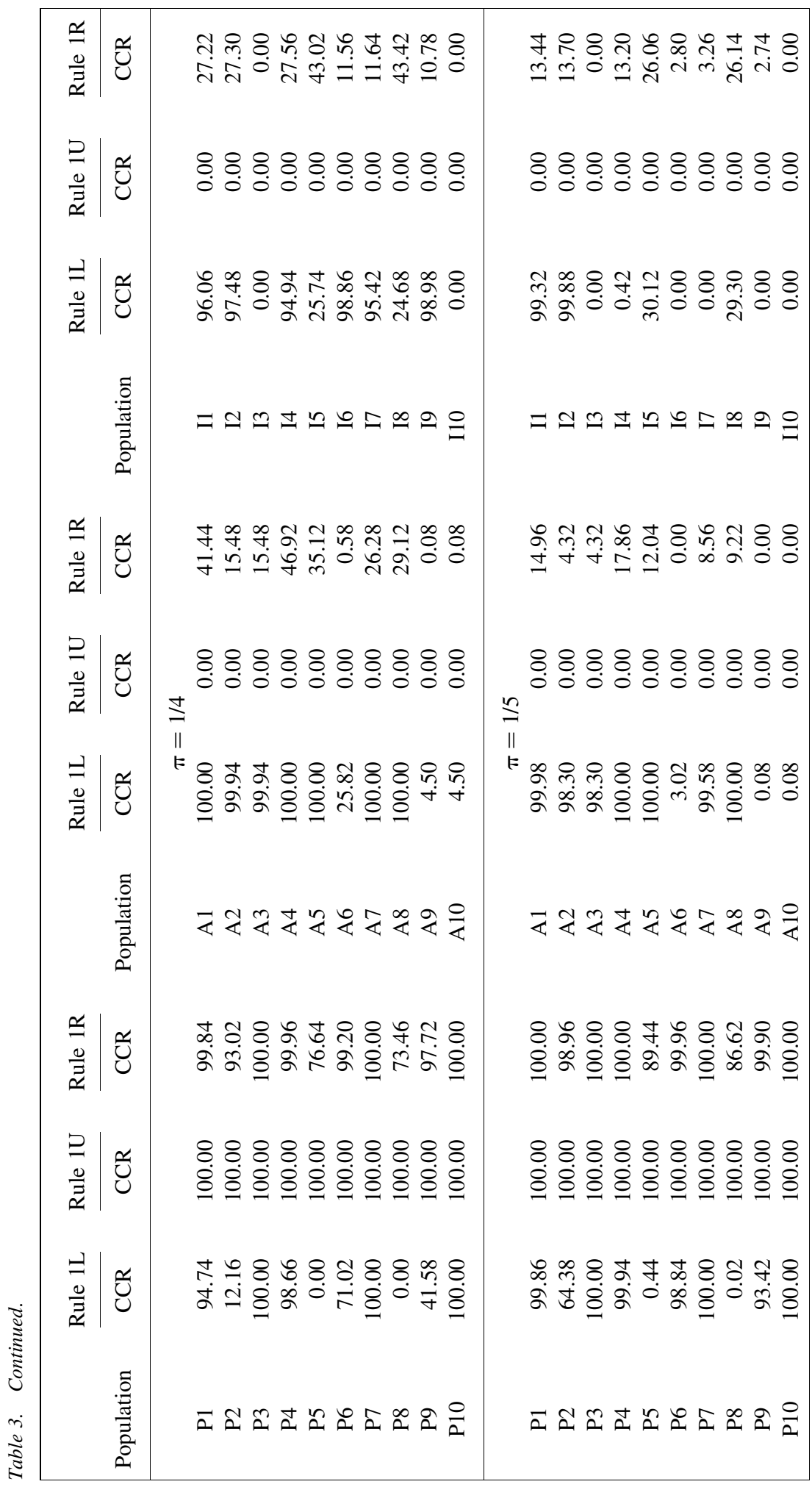




\begin{tabular}{|c|c|c|c|}
\hline 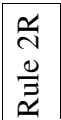 & 艺 & 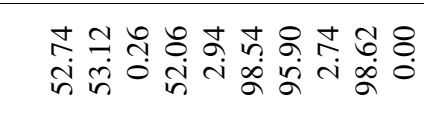 & 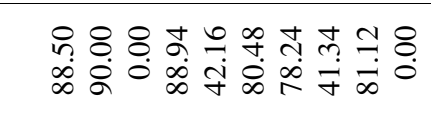 \\
\hline 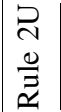 & Ư & 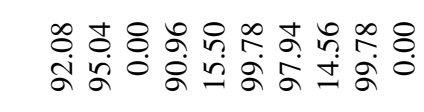 & 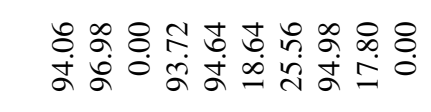 \\
\hline 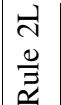 & 苻 & 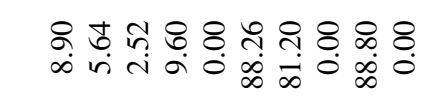 & 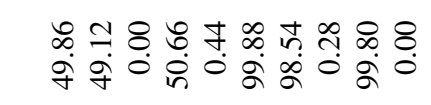 \\
\hline & 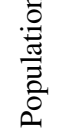 & $\Xi \simeq \cong \pm \curvearrowleft \varrho ニ \infty 9 \varrho$ & $\exists \simeq \cong \pm \curvearrowleft \varrho ニ \infty 9 \varrho$ \\
\hline 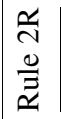 & 通 & 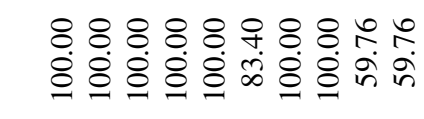 & 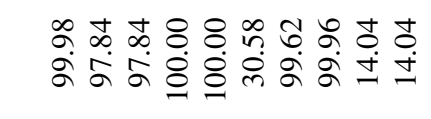 \\
\hline 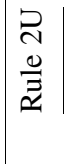 & 过 & 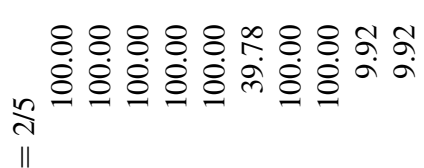 & 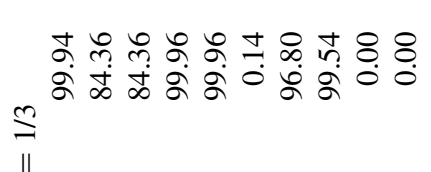 \\
\hline$\left|\begin{array}{l}\vec{A} \\
\stackrel{\vec{Z}}{\widetilde{\Xi}}\end{array}\right|$ & $\ddot{\widetilde{U}}$ & 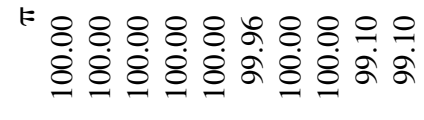 & 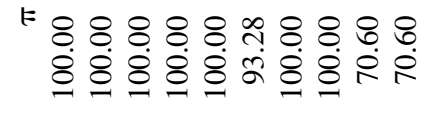 \\
\hline & 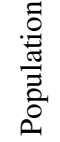 & 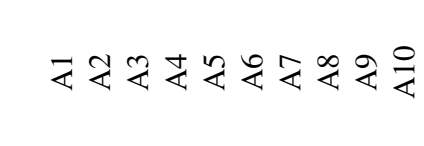 & 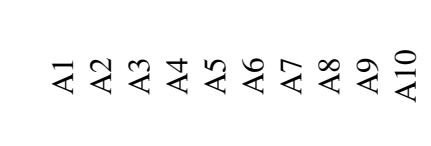 \\
\hline 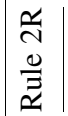 & Ư & 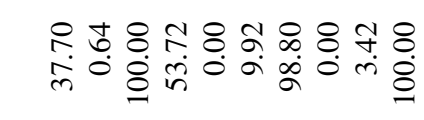 & 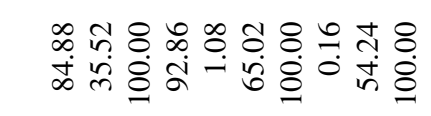 \\
\hline 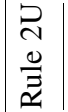 & 选 & 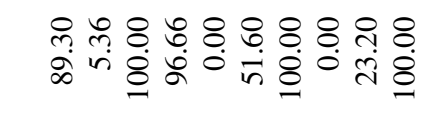 & 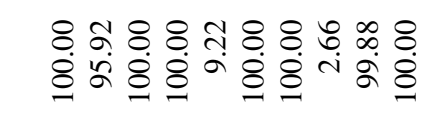 \\
\hline 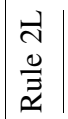 & Ư & 市 \& \& & 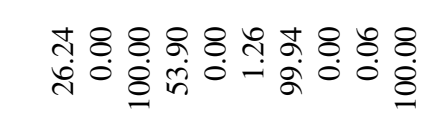 \\
\hline & 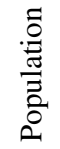 & 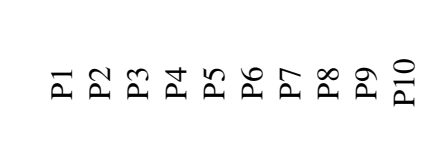 & 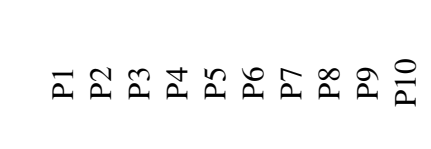 \\
\hline
\end{tabular}




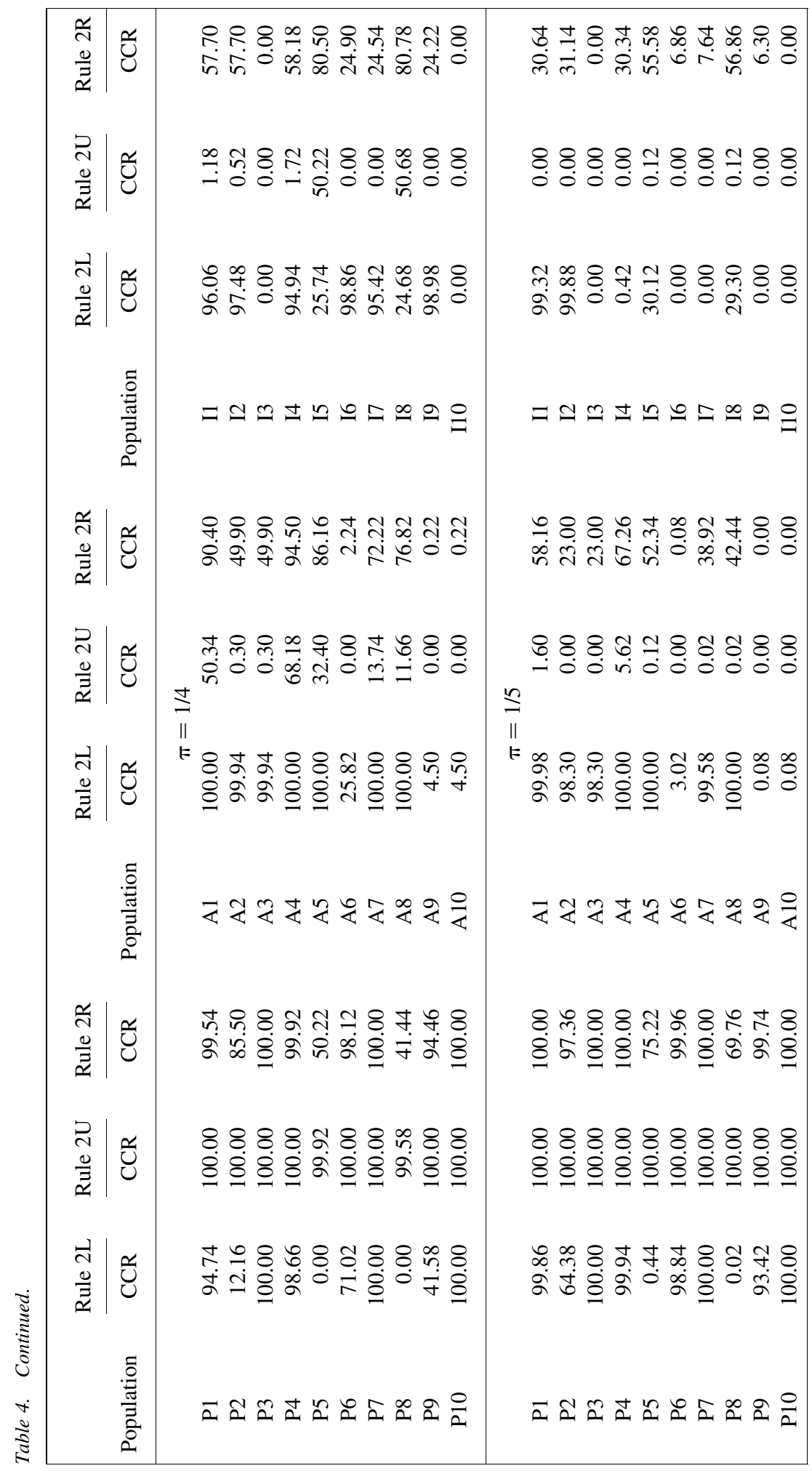




\begin{tabular}{|c|c|c|c|}
\hline 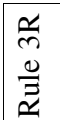 & 웜 & 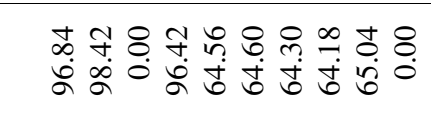 & 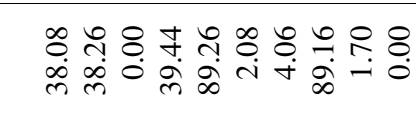 \\
\hline $\mid \begin{array}{l}2 \\
0 \\
\frac{0}{Z} \\
\approx\end{array}$ & 芑 & 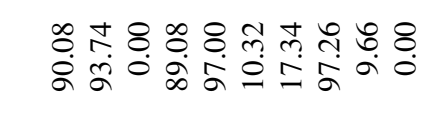 & 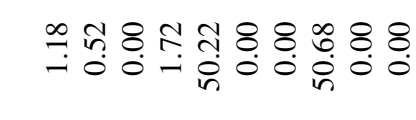 \\
\hline 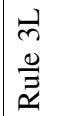 & 苻 & 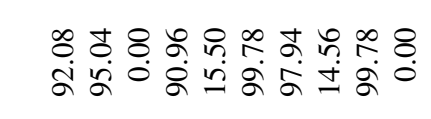 & 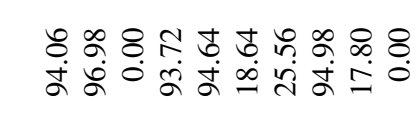 \\
\hline & 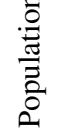 & 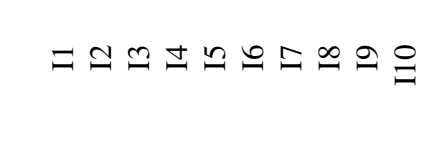 & $=\simeq \cong \pm \curvearrowleft \varrho ニ \infty \Omega \varrho$ \\
\hline $\begin{array}{l}\frac{\sigma}{m} \\
\frac{0}{\vec{z}} \\
\underline{2}\end{array}$ & 苻 & 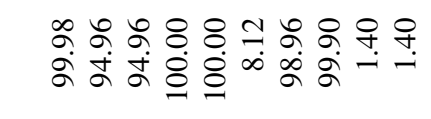 & 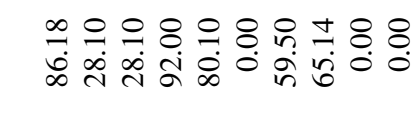 \\
\hline $\begin{array}{l}2 \\
\infty \\
\frac{0}{2} \\
\propto 2\end{array}$ & 苻 & 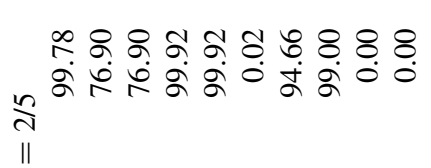 & 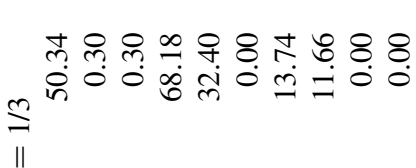 \\
\hline $\begin{array}{l}\vec{m} \\
\stackrel{0}{\Xi} \\
\approx\end{array}$ & 苻 & 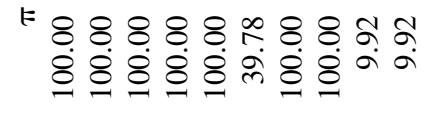 & 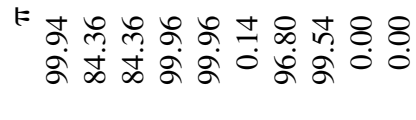 \\
\hline & 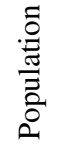 & 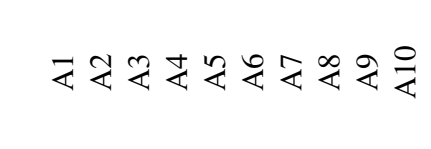 & 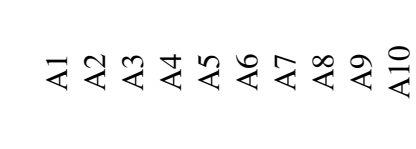 \\
\hline $\begin{array}{l}\frac{a}{m} \\
\frac{0}{\tilde{m}}\end{array}$ & 苻 & 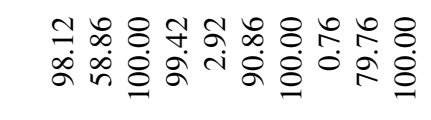 & 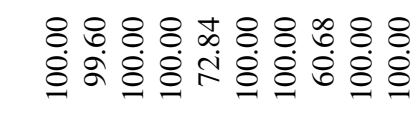 \\
\hline 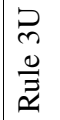 & $\stackrel{\overbrace{}}{0}$ & 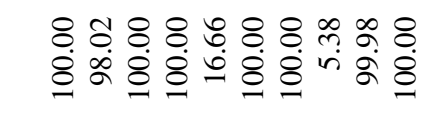 & 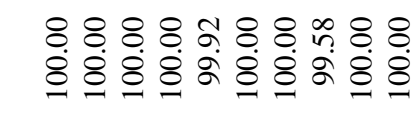 \\
\hline $\begin{array}{l}\stackrel{m}{m} \\
\frac{0}{\Xi}\end{array}$ & 苻 & 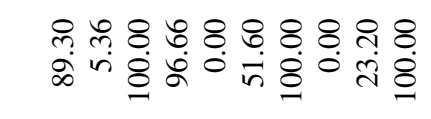 & 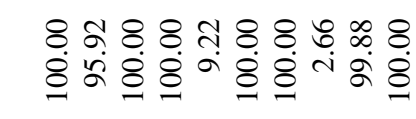 \\
\hline & 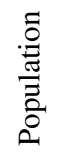 & 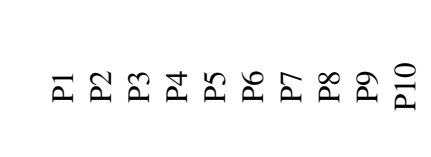 & 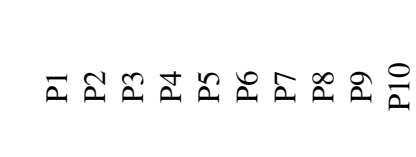 \\
\hline
\end{tabular}




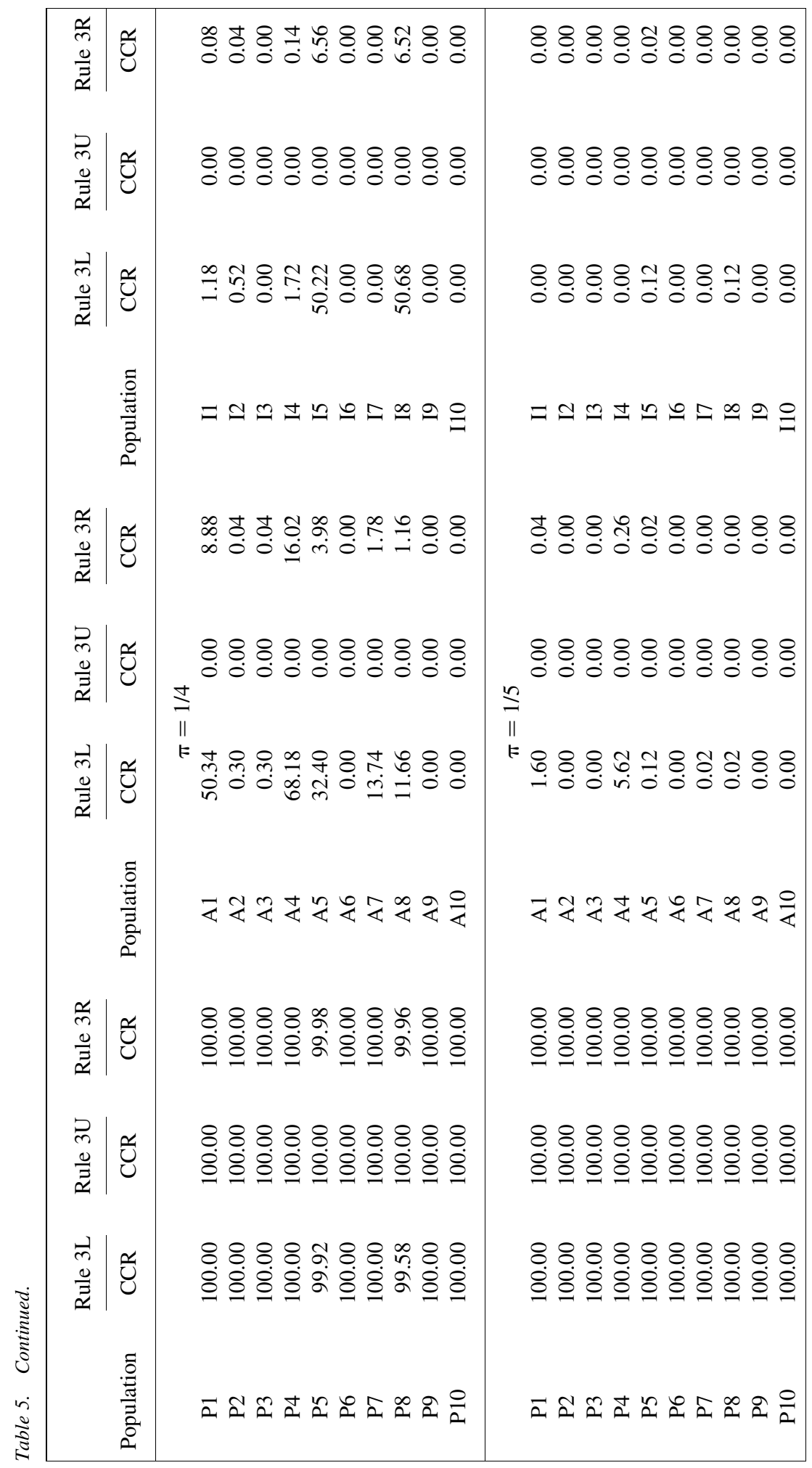



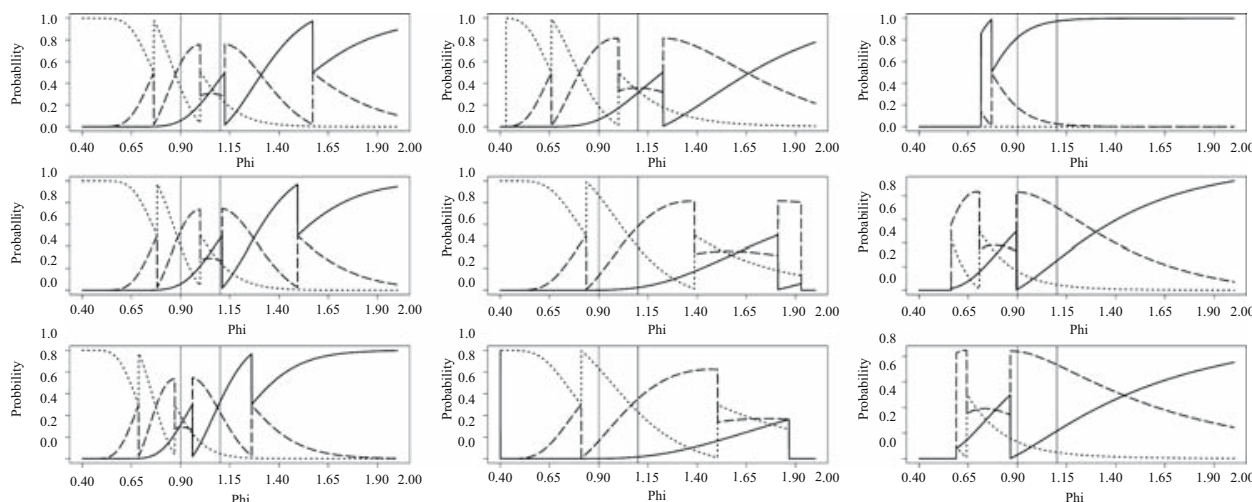

Fig. 1. Graphical comparison of the probabilities of recapture proneness, recapture aversion \& listindependence under the Rule $2 R$, associated with the Proposal 2, with $\pi=1 / 3$ over different $\phi$ values. Continuous, dotted and longdash lines respectively refer the probabilities corresponding to recapture proneness, recapture aversion and list-independence.

\section{Real Data Illustration}

In this section, we illustrate the classification strategies formulated under the two selected proposals (i.e., Proposals 1 and 2) described in Section 2 through the application on three real data sets from three different fields - demography, crime statistics and social sciences.

Firstly, we consider the Malawi death data obtained from a Population Change Survey to estimate birth, death and migration rates conducted by the National Statistical Office in Malawi between 1970 and 1972. Greenfield (1975) introduced this data set. Later, it has also been used by Nour (1982) and Chatterjee and Mukherjee (2016c). Very large values of $\hat{c}$ for all the strata, except Lilongwe, clearly indicate recapture proneness. Thus, we consider the data for Lilongwe and Other Urban Areas (see top panel of Table 6) for comparative analysis of the proposed classification rules.

Secondly, we use Homicide data, which are analyzed by Eckberg (2000) to establish the utility of dual enumeration methods for estimating the total number of unrecorded murders in South Carolina, 1877-1878. This interesting work was meant for tracing the historic trends in homicide based on the two sources alone and the author claimed that the popular

Table 6. Three real data sets in DRS format which are used for the classification analysis.

\begin{tabular}{|c|c|c|c|c|c|}
\hline \multirow[b]{3}{*}{ Data } & \multirow[b]{3}{*}{ Populations } & \multicolumn{3}{|c|}{ Count } & \multirow{3}{*}{$\frac{\text { Estimate of } c}{\hat{c}}$} \\
\hline & & \multirow{2}{*}{$\frac{\text { List } 1}{x_{1} .}$} & \multirow{2}{*}{$\frac{\text { List } 2}{x \cdot 1}$} & \multirow{2}{*}{$\frac{\text { Matched }}{x_{11}}$} & \\
\hline & & & & & \\
\hline \multirow[t]{2}{*}{ Malawi death } & Lilongwe & 324 & 216 & 192 & 0.593 \\
\hline & Other Urban Areas & 1960 & 2450 & 1645 & 0.839 \\
\hline \multirow[t]{3}{*}{ Homicide* $^{*}$} & Zero-Two & 29 & 102 & 23 & 0.793 \\
\hline & Three-Five & 56 & 74 & 42 & 0.750 \\
\hline & Six & 50 & 43 & 32 & 0.640 \\
\hline \multirow[t]{2}{*}{ Handloom } & Ward No. 2 & 126 & 107 & 85 & 0.675 \\
\hline & Ward No. 16 & 131 & 103 & 50 & 0.382 \\
\hline
\end{tabular}

*Each of the three populations belong to this data set are named in terms of holding index score. 
ChandraSekar-Deming estimator would face a formidable undercount problem. This happens due to a possible positive correlation between two data sources - $(i)$ South Carolina Department of Archives and History and (ii) News and Courier Reports. Dual system data were available for all 33 counties in South Carolina. Following the proposal of ChandraSekar and Deming (1949), all counties (except Charleston) are divided into three homogeneous groups based on a $0-6$ point index scale that measures the thoroughness of county archives. For more details on the data source and index scaling, readers are referred to Eckberg (2000, 5-9). Data in DRS form are presented in the middle panel of Table 6.

In addition to the above two data sets, we consider another data set on handloom workers. This new data are from a survey aimed to estimate the undercount in the census of handloom workers (master weavers and labours only) attached to the handloom industry residing at Gangarampur in South Dinajpur district of the state West Bengal, India in 2013. Handloom products have a rich tradition in this state and the handloom industry occupies a place second only to agriculture in providing livelihood to the people. In the urban area of Gangarampur, there are sixteen wards; two of these wards (Wards No. 2 and 16) are selected for Post Enumeration Survey (PES) to evaluate the coverage in the original census (SOSU 2014). Surprisingly, the nature of the data on these two wards are found to be different in terms of recapture proportion. Since this counting task is meant for the benefit of the workers attached to the handloom industry, a general thought is that the census and the PES might be positively related. On the other hand, some people may consider that one time enrollment is enough. So, if one is counted at the time of census, he/she may be reluctant at the time of the PES and that implies $\phi$ to be less than 1. Thus, in both of the possibilities, the ChandraSekar-Deming estimator would not be appropriate. Surveyors reported that workers in Ward No. 16, which is very close to the town center, might be somewhat reluctant to enlist themselves a second time (i.e., at the time of the PES). Moreover, most of them work outside (other districts) and usually come home during particular seasons. That is why Ward No. 16 shows in very low matches compared to Ward No. 2. Moreover, the beliefs of the experts of the Textile Directorate of the Government of West Bengal also drive the idea that the ChandraSekar-Deming estimates (157 and 270 respectively) fail to extract the sizes with precision. However, they expect that the ChandraSekar-Deming method yields a slight undercount for Ward No. 2 and a high overcount for Ward No. 16. The bottom panel of Table 6 presents the DRS data for these two wards.

Table 6 presents the list-wise counts and matched records for each of the three data sets mentioned above. In addition, we also present values of the key statistic $\hat{c}=\left(x_{11} / \mathrm{x}_{1}\right.$.) for each data set in Table 6 . Results on the directional classification for the above three data sets are presented in Table 7. In addition, estimates of the probabilities associated with the directional classifications found are presented for each of the seven real populations in Table 7. These estimated probabilities can be treated as a measure of chance (or uncertainty) behind the directional classifications found.

In the light of the findings from the selected strategies (under proposals 1 and 2 with $\pi=(1 / 3,1 / 4)$, the populations Lilongwe and Other Urban Areas are classified as listindependent and recapture prone, respectively. The classification result for Lilongwe is quite interesting, as it indicates a difference from the conventional assumption of positive dependence in DRS on demographic application. All the populations under the Homicide data set exhibit recapture proneness except for the holding index score 6 , for which rules $\mathrm{L}$ 


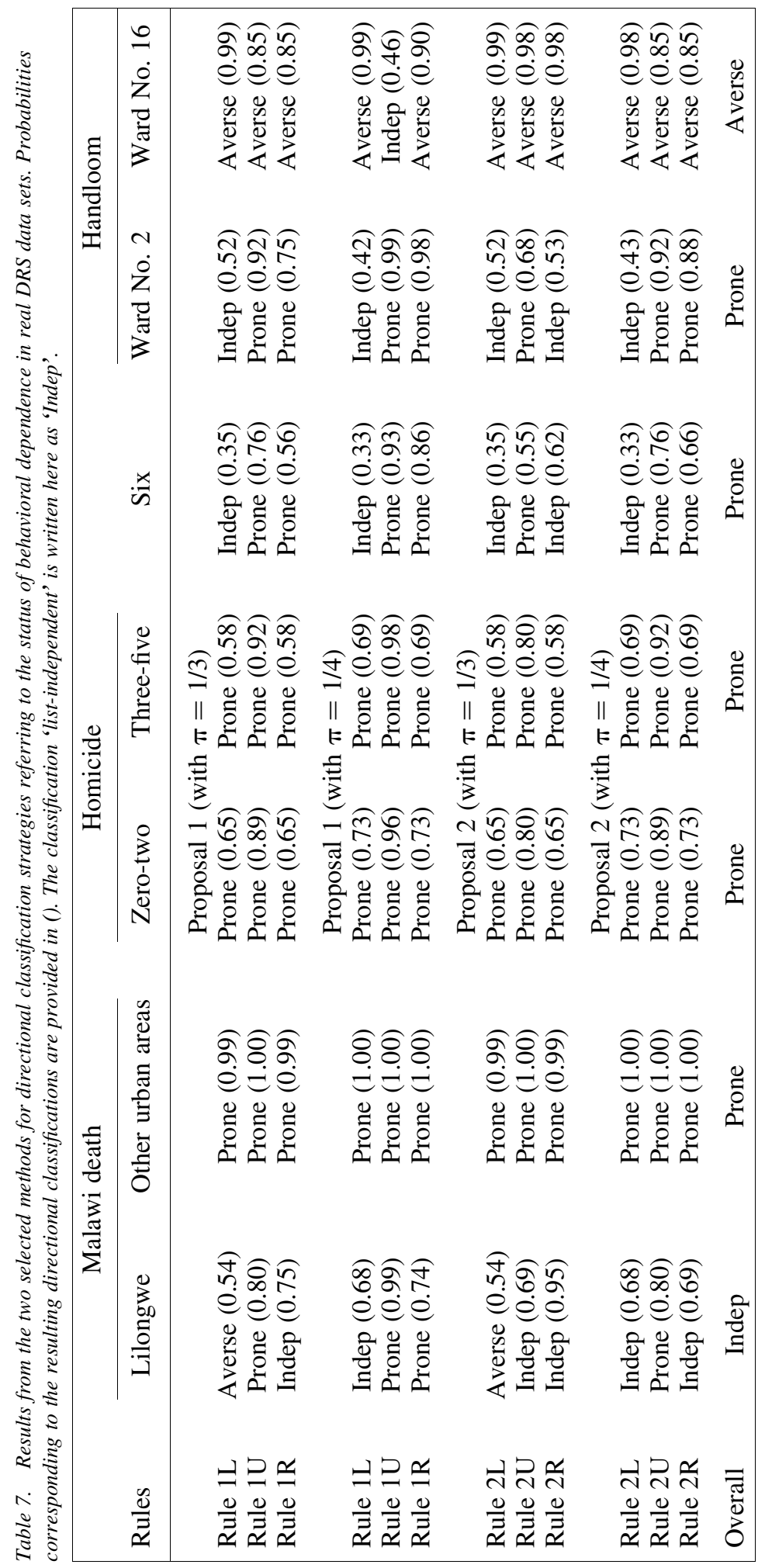


find no dependence. For Ward No. 16 under the handloom data set, the strategies agree and classify the ward as recapture averse. However, inference for the population in Ward No. 2 is in favor of recapture proneness. Indeed, in the conflicting cases, we recommend going with the classification made by the randomized Rule $2 R$, as Proposal 2 along with randomized rule $(\mathrm{R})$ is found to be better in the simulation based comparative study performed earlier. In the last row of Table 7, we present the overall classification status of the population in each real data set and that is found to be identical with the inference from Rule $2 R$.

It is to be noted that inference about the direction of possible behavioral dependence in any data set drawn in literature may not match with the conclusions of the classification strategies prescribed in this present article.

\section{Conclusion}

From the extensive literature on capture-recapture data analysis on human population, it is quite clear that assumption of list-independence does not hold satisfactorily in many instances. As far as homogeneous human population size estimation is concerned, twosample capture-recapture experiments are very common and the model $M_{t b}$ is best suited. However, this model seriously suffers from the non-identifiability problem and analyses in the literature suggest that the availability of knowledge on the direction of behavioral dependency could improve the inference to a great extent. Eliciting such information is crucial in DRS. To address this issue, we develop several comparable strategies for classification of the given population in terms of the direction of dependence (i.e., whether the given population is recapture prone or averse) under a mild and realistic assumption. Among the three proposals on bounds for unknown $\phi$, Proposal 2 is found to be better suited. On the other hand, the classification strategy based on randomized technique (R) is quite appealing for the development of more efficient inference in the context of $M_{t b}$-DRS. Hence, the strategy, Rule $2 R$, is quite accurate except for particular situations with small recapture probabilities. In real life applications, this strategy provides us with a useful tool for classifying human populations.

With the knowledge inferred in this article on the direction of dependence in DRS, model $M_{t b}$ can be successfully analyzed in the Bayesian paradigm. For example, Chatterjee and Mukherjee (2016c, 2018) have shown that availability of knowledge on the direction of behavioral dependence helps to gain precision in the estimation of population size. One can also analogously improve the Bayesian methodology proposed in Lee and Chen (1998) and Lee et al. (2003) by choosing suitable priors using our strategies. Also, inference on population size assuming any particular dependence type (i.e., recapture prone or averse) can be improved based on bound or inequality. For example, in Greenfield (1975) and Nour (1982), recapture proneness is assumed for demographic data. Their assumption can be verified using our methodology. For both of these approaches, the knowledge on the possible direction of dependence gained from the methods proposed in our present article results in more precise inference. Extension of the proposed behavioral classification strategies may be possible for more than two capture occasions.

Throughout the article, we assume that ordering of the two samples (or lists) in DRS are known, that is, one list is completely prepared before the other. However in some cases, 
listing by two sources are either parallel or their ordering is not known. In such cases, consider a typical situation where the number of recaptured individuals $\left(x_{11}\right)$ is small compared to the number of one-time captured individuals, that is $x_{10}$ or $x_{01}$. Then, our proposed classification results may differ if ordering of the lists is interchanged as observed in the case of Homicide data, with holding index score Zero-Two. In this case, reversal of ordering for the lists will lead to a conclusion of recapture aversion instead of proneness. As per the definition of grouping, these data exhibit lack of thoroughness of the county archives and hence, $x_{11}$ is small compared to $x_{10}$ or $x_{01}$.

\section{Appendix}

\subsection{Proof of Theorem 1}

Firstly, $\operatorname{Pr}(R P)=\operatorname{Pr}\left(X_{P}=1\right)$. Therfore,

$$
\begin{aligned}
\operatorname{Pr}(R P) & =\operatorname{Pr}\left(\frac{2 \hat{c}}{1+\pi}>\phi_{u}\right)+\delta_{p} \operatorname{Pr}\left(\frac{2 \hat{c}}{1+\pi} \leq \phi_{u}<\hat{c} \pi^{-1 / 2}\right) \\
& =1-F\left(\frac{(1+\pi) \phi_{u}}{2}\right)+\delta_{p}\left[F\left(\frac{(1+\pi) \phi_{u}}{2}\right)-F\left(\phi_{u} \pi^{1 / 2}\right)\right] \\
& =1-\left[\delta_{p} F\left(\phi_{u} \pi^{1 / 2}\right)+\left(1-\delta_{p}\right) F\left(\frac{(1+\pi) \phi_{u}}{2}\right)\right]
\end{aligned}
$$

where $F(x)$ denoted as the cumulative distribution function of the normal variate $\hat{c}$ at $x$ such that $\hat{c}$ has mean $c$ and variance equals to $\sigma_{\hat{c}}^{2}=V(\hat{c})$. This distribution for $\hat{c}$ is asymptotic. Thus, $F(x)=\Phi\left(\frac{x-c}{\sigma_{\hat{c}}}\right)$, where $\Phi$ has its usual meaning, that is, cumulative distribution function of a standard normal variate.

Secondly, $\operatorname{Pr}(R A)=\operatorname{Pr}\left(X_{p}=0, X_{a}=1\right)$. Therefore,

$$
\begin{aligned}
\operatorname{Pr}(R A)= & \operatorname{Pr}\left(\hat{c} \pi^{-1 / 2}<\phi_{l}, X_{p}=0\right)+\delta_{a} \operatorname{Pr}\left(\frac{2 \hat{c}}{1+\pi} \leq \phi_{l} \leq \hat{c} \pi^{-1 / 2}, X_{p}=0\right) \\
= & \operatorname{Pr}\left(\hat{c} \pi^{-1 / 2}<\phi_{l}, \hat{c} \pi^{-1 / 2} \leq \phi_{u}\right)+\left(1-\delta_{p}\right) \operatorname{Pr}\left(\hat{c} \pi^{-1 / 2}<\phi_{l}, \frac{2 \hat{c}}{1+\pi} \leq \phi_{u}<\hat{c} \pi^{-1 / 2}\right) \\
& +\delta_{a}\left(1-\delta_{p}\right) \operatorname{Pr}\left(\frac{2 \hat{c}}{1+\pi} \leq \phi_{l}<\hat{c} \pi^{-1 / 2}, \frac{2 \hat{c}}{1+\pi} \leq \phi_{u}<\hat{c} \pi^{-1 / 2}\right) \\
& +\delta_{a} \operatorname{Pr}\left(\frac{2 \hat{c}}{1+\pi} \leq \phi_{l} \leq \hat{c} \pi^{-1 / 2}, \hat{c} \pi^{-1 / 2} \leq \phi_{u}\right) \\
= & \operatorname{Pr}\left(\hat{c} \pi^{-1 / 2}<\phi_{l}\right)+\left(1-\delta_{p}\right) \cdot 0+\delta_{a} \operatorname{Pr}\left[\phi_{l} \pi^{1 / 2} \leq \hat{c} \leq \min \left(\frac{(1+\pi) \phi_{l}}{2}, \phi_{u} \pi^{1 / 2}\right)\right] \\
& +\delta_{a}\left(1-\delta_{p}\right) \operatorname{Pr}\left(\phi_{u} \pi^{1 / 2}<\hat{c} \leq \frac{(1+\pi) \phi_{l}}{2}\right) .
\end{aligned}
$$

If $\phi_{l}$ and $\phi_{u}$ are chosen such that $\frac{(1+\pi) \phi_{l}}{2}<\phi_{u} \pi^{1 / 2}$, therefore,

$$
\operatorname{Pr}(R A)=\delta_{a} F\left(\frac{(1+\pi) \phi_{l}}{2}\right)+\left(1-\delta_{a}\right) F\left(\phi_{l} \pi^{1 / 2}\right)
$$


Lastly,

$$
\begin{aligned}
\operatorname{Pr}(L I)= & \operatorname{Pr}\left(X_{p}=0, X_{a}=0\right) \\
= & \operatorname{Pr}\left(X_{p}=0\right)-\operatorname{Pr}\left(X_{p}=0, X_{a}=1\right) \\
= & {\left[\delta_{p} F\left(\phi_{u} \pi^{1 / 2}\right)+\left(1-\delta_{p}\right) F\left(\frac{(1+\pi) \phi_{u}}{2}\right)\right] } \\
& -\left[\delta_{a} F\left(\frac{(1+\pi) \phi_{l}}{2}\right)+\left(1-\delta_{a}\right) F\left(\phi_{l} \pi^{1 / 2}\right)\right]
\end{aligned}
$$

Again, if $\frac{(1+\pi) \phi_{l}}{2}>\phi_{u} \pi^{1 / 2}$ holds,

$$
\begin{aligned}
\operatorname{Pr}(R A)= & F\left(\phi_{l} \pi^{1 / 2}\right)+\delta_{a}\left[F\left(\phi_{u} \pi^{1 / 2}\right)-F\left(\phi_{l} \pi^{1 / 2}\right)\right] \\
& +\delta_{a}\left(1-\delta_{p}\right)\left[F\left(\frac{(1+\pi) \phi_{l}}{2}\right)-F\left(\phi_{u} \pi^{1 / 2}\right)\right] \\
= & \left(1-\delta_{a}\right) F\left(\phi_{l} \pi^{1 / 2}\right)+\delta_{a}\left(1-\delta_{p}\right) F\left(\frac{(1+\pi) \phi_{l}}{2}\right)+\delta_{a} \delta_{p} F\left(\phi_{u} \pi^{1 / 2}\right)
\end{aligned}
$$

and similarly,

$$
\begin{aligned}
\operatorname{Pr}(L I)= & {\left[\delta_{p}\left(1-\delta_{a}\right) F\left(\phi_{u} \pi^{1 / 2}\right)+\left(1-\delta_{p}\right) F\left(\frac{(1+\pi) \phi_{u}}{2}\right)\right] } \\
& -\left[\delta_{a}\left(1-\delta_{p}\right) F\left(\frac{(1+\pi) \phi_{l}}{2}\right)+\left(1-\delta_{a}\right) F\left(\phi_{l} \pi^{1 / 2}\right)\right] .
\end{aligned}
$$

This completes the proof.

\subsection{Mathematical Proofs and Justification on the Two Roots of $\phi$}

The quadratic equation, corresponding to inequality (2), can be written as

$$
\phi^{2}+u \phi+v=0
$$

where $u=\frac{k x_{\cdot 1}-(\pi+1) x_{11}}{\pi x_{1} \text {. }}$ and $v=\hat{c}^{2} \pi^{-1}$. This quadratic equation (S1) has two roots, $\phi_{0}$ and $\phi_{1}$, which satisfy

$$
\phi_{0}+\phi_{1}=-u=\frac{(\pi+1) x_{11}-k x_{\cdot}}{\pi x_{1}} \text { and } \phi_{0} \phi_{1}=v=\hat{c}^{2} \pi^{-1} .
$$

The two roots $\phi_{0}$ and $\phi_{1}$ become real if and only if $u^{2}-4 v \geq 0$. In order for both roots to be strictly positive, further restriction $\phi_{0}+\phi_{1}>0$, equivalently $u<0$, is needed, since $\phi_{0} \phi_{1}=v>0$ as $x_{11}>0$. Thus, the inequality condition $u^{2}-4 v \geq 0$ is equivalent to $(-u) \geq 2 \sqrt{v}$ i.e., $u \leq-2 \sqrt{v}$. Now, the condition $u \leq-2 \sqrt{v}$ means

$$
k x_{\cdot 1}-(\pi+1) x_{11} \leq-2 x_{11} \sqrt{\pi} \Leftrightarrow k \leq \frac{x_{11}}{x_{\cdot 1}}(1-\sqrt{\pi})^{2}
$$

since $\hat{c}=\left(x_{11} / x_{1}\right)$. 
Hence, from the above, it is clear that the two roots $\phi_{0}$ and $\phi_{1}$ are functions of the only unknown $k$ and they satisfy $\phi_{0} \leq \phi \leq \phi_{1}$ in order to maintain the inequality (3). In particular, equality in the above equation (S2) holds only when $u^{2}=4 v$, or equivalently, $(-u) / 2=\sqrt{v}$ or equivalently, $\frac{\left(\phi_{0}+\phi_{1}\right)}{2}=\sqrt{\phi_{0} \phi_{1}}$ holds and this A.M. $=$ G.M. condition holds if and only if $\phi_{0}=\phi_{1}=\phi=\sqrt{v}=\hat{c} \pi^{-1 / 2}$. In addition, structurally $k \geq 0$ holds under the assumption of $p \geq \pi$. Hence, note that the upper bound (S2) on the non-negative term $k$ is the necessary and sufficient condition for both the roots $\phi_{0}$ and $\phi_{1}$ to be positive real-valued.

The two real positive roots, $\phi_{0}$ and $\phi_{1}$, of the Equation (S1) are

$$
\phi_{0}=\frac{1}{2}\left(-u-\sqrt{u^{2}-4 v}\right) \text { and } \phi_{1}=\frac{1}{2}\left(-u+\sqrt{u^{2}-4 v}\right),
$$

with the restrictions $u<0$ and $u^{2}-4 v \geq 0$, where $u$ and $v$ are stated in (S1). From the above expressions of $\phi_{0}$ and $\phi_{1}$, we have the values of $\phi_{0}$ and $\phi_{1}$ corresponding to the lower bound of $k$ (i.e., $k=0$ ) are $\hat{c}$ and $\hat{c} \pi^{-1}$ respectively. Similarly, when $k$ attains its upper bound (S2), both of $\phi_{0}$ and $\phi_{1}$ are equal to $\hat{c} \pi^{-1 / 2}$. This implies

$$
\hat{c} \leq \phi_{0} \leq \hat{c} \pi^{-1 / 2} \text { and } \hat{c} \pi^{-1 / 2} \leq \phi_{1} \leq \hat{c} \pi^{-1} .
$$

Now, let us examine the nature of the roots in terms of the unknown $k$.

$$
\frac{d \phi_{0}}{d k}=\frac{d \phi_{0}}{d u} \cdot \frac{d u}{d k}=-\frac{x_{\cdot}}{2 \pi x_{1} .}\left(1+\frac{u}{\sqrt{u^{2}-4 v}}\right) .
$$

Given the stated restrictions $u<0$ and $u^{2}-4 v \geq 0$,

$$
u^{2}-4 v<u^{2} \Rightarrow \sqrt{u^{2}-4 v}<-u \Rightarrow\left(1+\frac{u}{\sqrt{u^{2}-4 v}}\right)<0 \Rightarrow \frac{d \phi_{0}}{d k}>0
$$

since, $x_{\cdot 1}, x_{1} ., \pi>0$. Thus, the smaller root, $\phi_{0}$, is monotonically increasing in $k$. As the product of the two roots is a constant, that is, independent of $k$, the other root, $\phi_{1}$, is monotonically decreasing. As we already found, the values of $\phi_{0}$ and $\phi_{1}$ corresponding to the lower bound of $k$ are $\hat{c}$ and $\hat{c} \pi^{-1}$ respectively. Therefore, $\phi_{0}\left(\phi_{1}\right)$ increases (decreases) to $\hat{c} \pi^{-1 / 2}$ as $k$ increases to its upper bound in (S2) and this finding meets the result (S3). This completes the justifications.

\section{References}

Bell, W.R. 1993. "Using information from demographic analysis in post-enumeration survey estimation." Journal of the American Statistical Association 88: 1106-1118. DOI: http://dx.doi.org/10.2307/2290805.

Bohning, D., P.V.D. Heijden, and J. Bunge. 2017. Capture-Recapture Methods for the Social and Medical Sciences (1st edition). Boca Raton, FL: Chapman Hall CRC Interdisciplinary Statistics.

Brittain, S. and D. Bohning. 2009. "Estimators in capture-recapture studies with two sources." Advanced Statistical Analysis 93: 23-47. DOI: https://doi.org/10.1007/ s10182-008-0085-y. 
ChandraSekar, C. and W.E. Deming. 1949. "On a method of estimating birth and death rates and the extent of registration." Journal of the American Statistical Association 44: 101-115. DOI: https://doi.org/10.1080/01621459.1949.10483294.

Chao, A., W. Chu, and H.H. Chiu. 2000. "Capture-recapture when time and behavioral response affect capture probabilities.” Biometrics 56: 427-433. DOI: https://doi.org/ 10.1111/j.0006-341X.2000.00427.x.

Chao, A., P.K. Tsay, S.H. Lin, W.Y. Shau, and D.Y. Chao. 2001. "Tutorial in biostatistics: The application of capture-recapture models to epidemiological data." Statistics in Medicine 20: 3123-3157. DOI: https://doi.org/10.1002/sim.996.

Chatterjee, K. and D. Mukherjee. 2016a. "An improved estimator of omission rate for census count: with particular reference to India." Communication in Statistics: Theory and Methods 45: 1047-1162. DOI: https://doi.org/10.1080/03610926.2013.854911.

Chatterjee, K. and D. Mukherjee. 2016b. "An improved integrated likelihood population size estimation in dual-record system." Statistics \& Probability Letters 110: 146-154. DOI: https://doi.org/10.1016/j.spl.2015.12.019.

Chatterjee, K. and D. Mukherjee. 2016c. "On the estimation of homogeneous population size from a complex dual-record system." Journal of Statistical Computation and Simulation 86: 3562-3581. DOI: https://doi.org/10.1080/00949655.2016.1173695.

Chatterjee, K. and D. Mukherjee. 2018. "A new integrated likelihood for estimating population size in dependent dual-record system." Canadian Journal of Statistics 46: 577-592. DOI: https://doi.org/10.1002/cjs.11477.

Eckberg, D.L. 2000. "A capture-recapture approach to the estimation of hidden historical killings." Proceedings of the 1999 meeting of the 332 Homicide Research Working Group, Washington, DC: Federal Bureau of Investigation, this volume was edited by P.H. Blackman, V.L. Leggett, B.L. Olson, and J.P. Jarvis.

El-Khorazaty, M.N. 2000. "Dependent dual-record system estimation of number of events: a capture-mark-recapture strategy." Environmetrics 11: 435-448. DOI: https:// doi.org/10.1002/1099-095X(200007/08)11:4<435::AID-ENV427>3.0.CO;2-2.

Gerritse, S.C., B.F.M. Bakker, D. Zult, and P.G.M. Van-der-Heijden. 2017. The impact of linkage errors and erroneous captures on the population size estimator due to implied coverage 2017-16. Vol. 16. Statistics Netherlands. Available at: https://www.cbs.nl/ en-gb/background/2017/39/impact-of-linkage-errors-and-erroneous-captures (accessed February 2020).

Gosky, R. and S.K. Ghosh. 2011. "A comparative study of Bayes estimators of closed population size from capture-recapture data." Journal of Statistical Theory and Practice 5: 241-260. DOI: https://doi.org/10.1080/15598608.2011.10412027.

Granerod, J., S. Cousens, N.W.S. Davies, N.S. Crowcroft, and S.L. Thomas. 2013. "New estimates of incidence of encephalitis in England." Emerging Infectious Diseases 19: 1455-1462. DOI: https://doi.org/10.3201/eid1909.130064.

Greenfield, C.C. 1975. "On the estimation of a missing cell in a $2 \times 2$ contingency table." Journal of the Royal Statistical Society A 138: 51-61. DOI: https://doi.org/10.2307/ 2345249.

Griffin, R.A. 2014. "Potential uses of administrative records for triple system modeling for estimation of census coverage error in 2020.” Journal of Offcial Statistics 30: 177-189. DOI: https://doi.org/10.2478/jos-2014-0012. 
Iñigo, J., A. Arce, J.M. Martn-Moreno, R. Herruzo, E. Palenque, and F. Chaves. 2003. "Recent transmission of tuberculosis in Madrid: application of capture-recapture analysis to conventional and molecular epidemiology." International Journal of Epidemiology 32: 763-769. DOI: https://doi.org/10.1093/ije/dyg098.

Jarvis, S.N., P.J. Lowe, A. Avery, S. Levene, and R.M. Cormack. 2000. "Children are not goldfish-mark/recapture techniques and their application to injury data." Injury Prevention 6: 46-50. DOI: http://dx.doi.org/10.1136/ip.6.1.46.

Lee, S.M. and C.W.S. Chen. 1998. "Bayesian inference of population size for behavioral response models." Statistica Sinica 8: 1233-1247. Available at: http://www3.stat. sinica.edu.tw/statistica/j8n4/j8n414/j8n414.htm (accessed February 2020).

Lee, S.M., W. Hwang, and L. Huang. 2003. "Bayes estimation of population size from capture-recapture models with time variation and behavior response." Statistica Sinica 13: 477-494. Available at: http://www3.stat.sinica.edu.tw/statistica/j13n2/j13n213/ j13n213.html (accessed February 2020).

Nour, E.-S. 1982. "On the estimation of the total number of vital events with data from dual collection systems." Journal of the Royal Statistical Society, Series A 145: 106-116. DOI: http://dx.doi.org/10.2307/2981424.

O'Connell, M. and K.H. Pollock. 1992. Wildlife 2001: Populations. Dordrecht: Springer.

Otis, D.L., K.P. Burnham, G.C. White, and D.R. Anderson. 1978. "Statistical inference from capture data on closed animal populations." Wildlife Monographs: A Publication of Wildlife Society 62: 3-135. Available at: https://pubs.er.usgs.gov/publication/ 70119899 (accessed February 2020).

Ruiz, M.S., A. O'Rourke, and S.T. Allen. 2016. "Using capture-recapture methods to estimate the population of people who inject drugs in Washington DC." AIDS and Behavior 20: 363-368. DOI: http://dx.doi.org/10.1007/s10461-015-1085-z.

SOSU, 2014. Report on the project survey of looms and work sheds in comprehensive handloom development programme in Dakshin Dinajpur District. Sampling and Offcial Statistics Unit, Indian Statistical Institute, Commissioned by: Directorate of Textiles, Government of West Bengal.

Wolter, K.M. 1986. "Some coverage error models for census data." Journal of the American Statistical Association 81: 338-346. DOI: http://dx.doi.org/10.2307/ 2289222.

Received November 2017

Revised August 2019

Accepted November 2019 